\title{
Collisions between tunable halo dimers: exploring an elementary four-body process with identical bosons
}

\author{
F. Ferlaino, ${ }^{1}$ S. Knoop, ${ }^{1}$ M. Mark, ${ }^{1}$ M. Berninger ${ }^{1}$ H. Schöbel,${ }^{1}$ H.-C. Nägerl, ${ }^{1}$ and R. Grimm ${ }^{1,2}$ \\ ${ }^{1}$ Institut für Experimentalphysik and Zentrum für Quantenphysik, Universität Innsbruck, 6020 Innsbruck, Austria \\ ${ }^{2}$ Institut für Quantenoptik und Quanteninformation, Österreichische Akademie der Wissenschaften, 6020 Innsbruck, Austria
}

(Dated: October 26, 2018)

\begin{abstract}
We study inelastic collisions in a pure, trapped sample of Feshbach molecules made of bosonic cesium atoms in the quantum halo regime. We measure the relaxation rate coefficient for decay to lower-lying molecular states and study the dependence on scattering length and temperature. We identify a pronounced loss minimum with varying scattering length along with a further suppression of loss with decreasing temperature. Our observations provide insight into the physics of a few-body quantum system that consists of four identical bosons at large values of the two-body scattering length.

PACS numbers: 21.45.-v, 33.15.-e, 34.50.Cx, 37.10.Pq
\end{abstract}

Few-body quantum systems in halo states exhibit unique properties [1]. A quantum halo is a very weakly bound state whose wave function extends far into the classically forbidden range. Halo systems are much larger than one would expect from the characteristic interaction range of their constituents. Many examples for halo states are known in nuclear physics, with the deuteron being a prominent example [2]. In molecular physics, the He dimer has for many years served as the prime example of a halo state [3, 4].

A particular motivation to study halo states is given by the concept of universality in few-body systems [5]. Since, in a halo state, short-range details of the interaction become irrelevant, the system is described by very few parameters and shows universal behavior in its low-energy observables. A halo dimer is the elementary two-body halo system. Here the only relevant length scale is given by the scattering length $a$, which describes the $s$-wave interaction between its two constituents. The size of the halo dimer is directly related to $a$ and the binding energy is $E_{b}=\hbar^{2} /\left(2 \mu a^{2}\right)$, where $\mu$ is the reduced mass. For three-body halo systems, universal Efimov states can exist [6, 7]. Here one additional parameter is required to fully describe the system in the universal limit. A natural further step is to investigate the universal physics of systems composed of four identical bosons. The fundamental properties of such systems are unexplored terrain, with the existence of an additional four-body parameter [8, 9], the scaling and threshold behavior, and the binding energies of four body-states [9] being open issues.

In the field of ultracold gases, the Feshbach association technique [10] has provided experimentalists with unprecedented possibilities to create and study halo dimers. In the case of certain Feshbach resonances, a considerable range of universality exists, where halo dimers can be conveniently controlled by a magnetic bias field to vary their binding energy and size. Such tunable halo dimers are unique probes to explore quantum phenomena related to universality. A binary collision between two halo dimers can be seen as an elementary four-body process. For a special kind of halo dimers such a four-body process has already attracted considerable atten- tion: halo dimers made of fermionic atoms in different spin states allow to create molecular Bose-Einstein condensates and to study the crossover to a fermionic superfluid [11]. Here a key point is the Pauli suppression effect that, in combination with the halo nature of the dimer, leads to stability against decay into lower-lying molecular states and favors elastic processes [12].

In this Letter, we study binary collisions in a pure, trapped sample of tunable halo dimers made of bosonic atoms. Halo dimers of this class have so far received much less experimental attention than their fermionic counterparts, although they represent an important link to universal few-body phenomena in systems of few interacting bosons; for three particles, an early example is the predicted atom-dimer "Efimov" resonances [13]. Halo dimers of bosonic atoms have been realized in ultracold gases of ${ }^{85} \mathrm{Rb}$ and ${ }^{133} \mathrm{Cs}$ [14, 15] and properties of the individual dimers, like binding energies, magnetic moments, and spontaneous decay rates, have been measured. In contrast, their collision properties have remained unexplored terrain. Because of the absence of a Pauli suppression effect, substantial inelastic decay to lower-lying molecular states can be expected. The observation of loss serves as a probe for dimer-dimer interactions [1, 10].

Our experiments are performed with ${ }^{133} \mathrm{Cs}$ atoms, which represent an excellent system to study few-body physics with bosons at large scattering lengths [7] because of the unique scattering properties [16]. In the lowest spin state at low magnetic fields, one finds a broad entrance-channel dominated $s$ wave Feshbach resonance [10, 17] along with an extraordinarily large background scattering length. The scattering properties of ultracold atoms are governed by the last bound $s$-wave state below the dissociation threshold as displayed in Fig. 1. In a wide magnetic-field range, this state carries a quantum halo character, where the two-body scattering length far exceeds the classical interaction range of the van der Waals potential, $r_{\mathrm{vdW}}\left(\approx 100 a_{0}\right)$, and the binding energy $E_{b}$ is much smaller than the corresponding $E_{\mathrm{vdW}}(\approx h \times 2.7 \mathrm{MHz})[18]$. Here $a_{0}$ is Bohr's radius.

Our experimental procedure to produce an optically trapped 

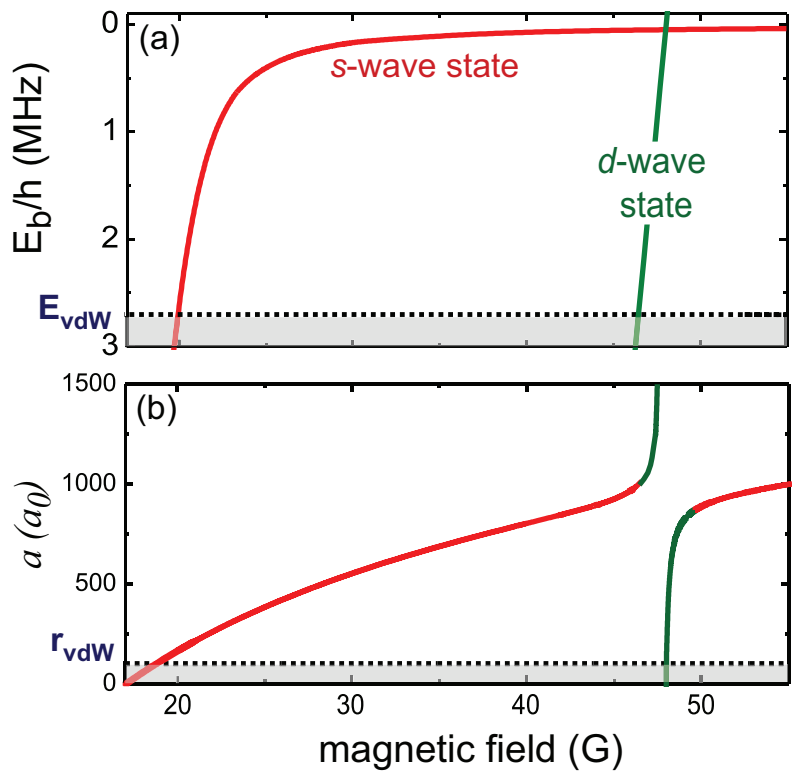

FIG. 1: (color online) Weakly bound $\mathrm{Cs}_{2}$ molecular states at low magnetic fields. (a) Binding energies of the relevant $\mathrm{Cs}_{2}$ dimer states. The zero-energy level is the $s$-wave threshold of two colliding Cs atoms in their absolute hyperfine ground state sub-level. (b) $s$-wave scattering length $a$ versus the magnetic field (see text). The shaded regions in (a) and (b) indicate the non-halo regime with $E_{b}>E_{\mathrm{vdW}}$, and $a<r_{\mathrm{vdW}}$, respectively.

sample of tunable halo dimers involves several stages. We initially prepare ultracold trapped ${ }^{133} \mathrm{Cs}$ atoms in their absolute hyperfine ground state sublevel $\left|F, m_{F}\right\rangle=|3,3\rangle$, similarly to Ref. [15]. The atoms are optically trapped by two crossed 1064-nm laser beams with waists of about $250 \mu \mathrm{m}$ and 36 $\mu \mathrm{m}$, while a magnetic field levitates the atoms against gravity [17, 19]. The levitation field ensures an optimized evaporative cooling of the atoms, which is realized by lowering the optical power in the trapping beams. We stop the cooling just before the onset of Bose-Einstein condensation to avoid too high atomic densities. At our lowest temperature of $20 \mathrm{nK}$, we obtain about $1.5 \times 10^{5}$ non-condensed atoms.

In the next stage, we create the halo dimers by Feshbach association. Here, the application of the levitation field is not appropriate since atoms and dimers in general have different magnetic moments. This leads to special requirements for the trap design. On the one hand, we need a sufficiently high optical gradient in the vertical direction to hold the atoms and dimers against gravity. On the other hand, we want to avoid the high density of a tight trap, which causes fast losses driven by atom-dimer collisions. These two requirements can be simultaneously fulfilled by using an elliptic trap potential with weak horizontal confinement and tight confinement in the vertical direction. Shortly before molecule production, we adiabatically convert the levitated trap to a non-levitated trap by simultaneously changing the levitation field, the optical power, and the trap ellipticity. The latter is modified by a rapid spatial oscillation of the $36-\mu \mathrm{m}$ waist beam in the hor-
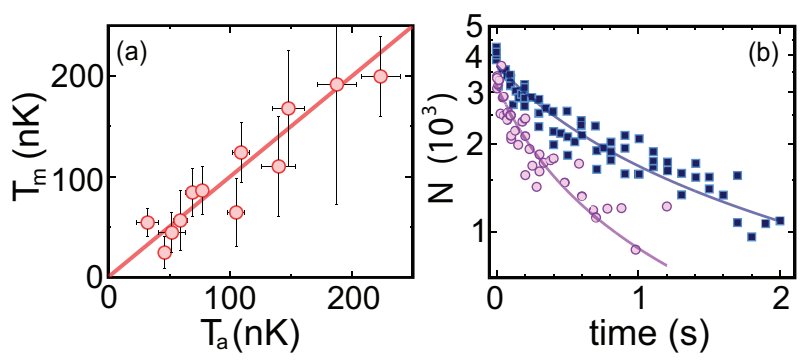

FIG. 2: (color online) Measurements on the trapped molecular sample. (a) Comparison between the atomic and molecular temperatures $T_{\mathrm{a}}$ and $T_{\mathrm{m}}$ at $35 \mathrm{G}$, where $E_{\mathrm{b}} \simeq h \times 87 \mathrm{kHz}$. The solid line indicates equal temperatures for atoms and molecules. (b) Number of halo dimers $N$ as a function of the holding time in trap at $28.3 \mathrm{G}$ ( $a=500 a_{0}$, squares) and at $45.6 \mathrm{G}\left(a=900 a_{0}\right.$, circles). The solid lines are fits to the data (see text).

izontal plane with the use of an acousto-optic modulator at a frequency of about $100 \mathrm{kHz}$, which greatly exceeds the typical trap frequencies, and thus creates a time-averaged optical potential [20, 21, 22]. The adiabatic change of the trap shape is set in a way to keep the peak density, the temperature, and thus the phase-space density constant [23]. Right after converting the trap, we associate the halo dimers by sweeping the magnetic field across the $200 \mathrm{mG}$ wide $d$-wave Feshbach resonance located at approximately $48 \mathrm{G}$ [15], (see Fig.11).

The molecular temperature can be set by adjusting the temperature of the initial atomic sample. To selectively measure the atomic and molecular temperatures, we spatially separate the two components with the Stern-Gerlach technique, and we perform a subsequent time-of-flight imaging, as described in Refs. [15, 19]. In Fig.2 (a), we compare the atomic and the molecular temperatures for a wide range of the initial temperatures. In spite of some overall heating in the conversion process, we observe that the dimers and the atoms have the same translational temperature in the trap. This observation may indicate an elastic interaction between atoms and dimers on the $10 \mathrm{~ms}$ time scale of our preparation sequence and it allows us to conveniently use the atomic sample to determine the temperature of the molecular gas.

In the last step of the preparation sequence, we selectively remove the atoms from the dipole trap. This is done by using a double-resonant purification scheme, which combines microwave excitation with resonant light [24], similarly to Ref. [25]. By absorption imaging after the Stern-Gerlach technique we verify that no atoms are remaining. We do not observe heating or loss of molecules as induced by the purification sequence.

We then use the pure, trapped sample of tunable halo dimers to study binary collisions. We measure inelastic decay, resulting from the relaxation into more deeply bound states. In such a process the conversion of internal into kinetic energy by far exceeds the trap depth and leads to immediate trap loss of all particles involved. All our experiments are carried out in a regime of very low temperatures $\left(k_{\mathrm{B}} T \ll E_{\mathrm{b}}\right)$, where the ini- 


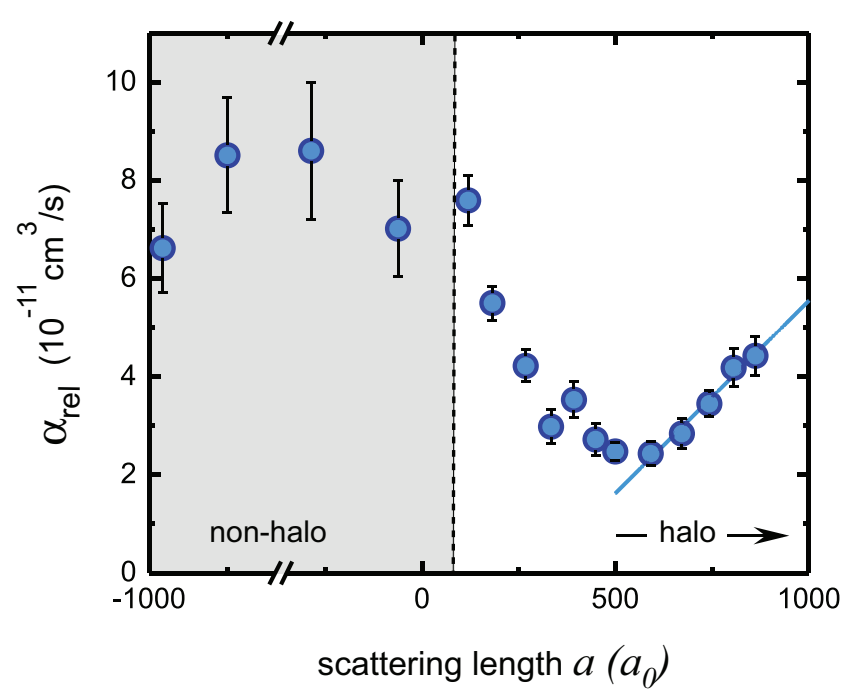

FIG. 3: (color online) Scattering length dependence of the relaxation rate coefficient $\alpha_{\text {rel }}$ at $120 \mathrm{nK}$. The solid line is a linear fit to the data in the region $a \geq 500 a_{0}$ (see text). The error bars refer to the statistical uncertainty. The shaded region indicates the $a<r_{\mathrm{vdW}}$ regime; here the experimental uncertainties are larger because the molecules have to be transferred through several avoided crossings with higher partial-wave states [15].

tial kinetic energy of the colliding dimers is not sufficient to break up the molecules, as observed for ${ }^{6} \mathrm{Li}$ halo dimers in Ref. [26]. Moreover, spontaneous dissociation observed for ${ }^{85} \mathrm{Rb}$ halo dimers [14, 27] is not possible as there is no energetically open channel. Other density-independent losses, such as background collisions or light-induced losses, can also be neglected under our experimental conditions. We can therefore completely attribute the observed losses to inelastic dimer-dimer collisions. The decay of the trapped dimer sample is thus described by the usual rate equation $\dot{N}=-\alpha_{\text {rel }} \bar{n} N$. Here $N$ indicates the number of dimers and $\alpha_{\text {rel }}$ the relaxation rate coefficient. The mean molecular density $\bar{n}$ is given by $\bar{n}=\left[m \bar{\omega}^{2} /\left(2 \pi k_{B} T\right)\right]^{3 / 2} N$ with $m$ being the atomic mass and $\bar{\omega}$ denoting the geometric mean of the trap frequencies.

We measure $\alpha_{\text {rel }}$ as a function of the scattering length $a$ for a fixed temperature $T=120 \mathrm{nK}$. We ramp the magnetic field to a desired value, and we then perform a lifetime measurement on the trapped dimers for storing times up to $2 \mathrm{~s}$. As an example, the time evolution of the dimer number in the optical trap is shown in Fig.2 (b) at two different values of the magnetic field. We observe the expected non-exponential decay of the dimer number. We extract the value of $\alpha_{\text {rel }}$ by fitting the data with the above rate equation. The lifetime measurements are then repeated at different values of the magnetic field in a range from $7 \mathrm{G}$ to $50 \mathrm{G}$.

The observed dependence of the relaxation rate coefficient on the scattering length reveals an interesting behavior; see Fig.3 Non-halo dimers exhibit a relative large and essentially constant collisional rate coefficient. In contrast, when the dimers enter the halo regime with increasing scattering length $\left(a>r_{\mathrm{vdW}}\right)$, the rate coefficient first drops to a minimum. The minimum is found at $a \approx 500 a_{0}\left(\sim 5 r_{\mathrm{vdW}}\right)$. For larger values of $a$, the rate coefficient increases with $a$. General considerations [28, 29, 30] suggest to first approximation a universal linear scaling law for $\alpha_{\text {rel }}$ according to $\alpha_{\text {rel }}=C(\hbar / m) a$ with a dimensionless constant $C$. From a linear fit for $a>500 a_{0}$ (solid line) we obtain an estimate for $C$ of about 3 .

An essentially constant relaxation rate coefficient for varying binding energy or magnetic field has been previously measured in collisions between non-halo dimers. Class of the nonhalo dimers includes ${ }^{23} \mathrm{Na}_{2}[31],{ }^{87} \mathrm{Rb}_{2}[32],{ }^{133} \mathrm{Cs}_{2}$ in various molecular states [33, 34], and also ${ }^{6} \mathrm{Li}_{2} p$-wave molecules as a process involving four identical fermions [35]. Our halo dimers composed of bosonic atoms thus show a novel and qualitatively different behavior.

In a second set of experiments, we study the temperature dependence of the relaxation rate coefficient. In the ultracold domain, inelastic two-body collision processes are usually described in terms of a simple rate constant, i.e. a rate coefficient being independent of the particular collision energy. This applies to the case of inelastic atom-atom collisions [36], as well as to collisions between deeply bound dimers [37, 38]. In contrast to this usual behavior, we find a strong temperature dependence of the loss rate coefficient of halo dimers, so that a simple rate constant model does not apply.

We have focused our measurements on the temperaturedependence in three different cases: the non-halo regime $\left(120 a_{0}\right)$, the loss minimum in the halo regime $\left(500 a_{0}\right)$, and a more extreme halo case with increased loss $\left(800 a_{0}\right)$. As shown in Fig.4, the dimers exhibit the expected constant relaxation rate outside of the halo regime $\left(\approx 9 \times 10^{-11} \mathrm{~cm}^{3} / \mathrm{s}\right)$. In the halo regime, we observe a clear decrease of the relaxation rate with decreasing temperature, both at $500 a_{0}$ and $800 a_{0}$, roughly following a $\sqrt{T}$-dependence. This surprising behavior raises the question whether the temperaturedependence of the relaxation rate is a property unique to halo states or whether it can also occur for other weakly bound Feshbach molecules. In the latter case, a halo dimer may just be seen as an extreme case of a weakly bound dimer. We speculate that this observation is related to the fact that collisions between weakly bound dimers can involve more complex processes which go beyond simple two-body mechanisms. For instance, the release of binding energy may lead to a fragmentation with three particles in the exit channel, i.e. a more deeply bound molecule and two free atoms. Breakup thresholds may manifest themselves in a more complicated dependence on the collision energy.

The possibility to suppress inelastic loss by controlling temperature and magnetic field leads to a favorable situation, in which halo dimers exhibit a high degree of stability. In particular, at $40 \mathrm{nK}$ and $500 a_{0}$, we measure a loss rate coefficient as low as $\sim 7 \times 10^{-12} \mathrm{~cm}^{3} / \mathrm{s}$. This unusually small value corresponds to an order of magnitude improvement in the stability against collisional decay with respect to previously investigated cases of ${ }^{23} \mathrm{Na}_{2}[31]$ and ${ }^{87} \mathrm{Rb}_{2}$ [32].

To conclude, we have studied inelastic collisions between tunable halo dimers composed of bosonic atoms. We have 


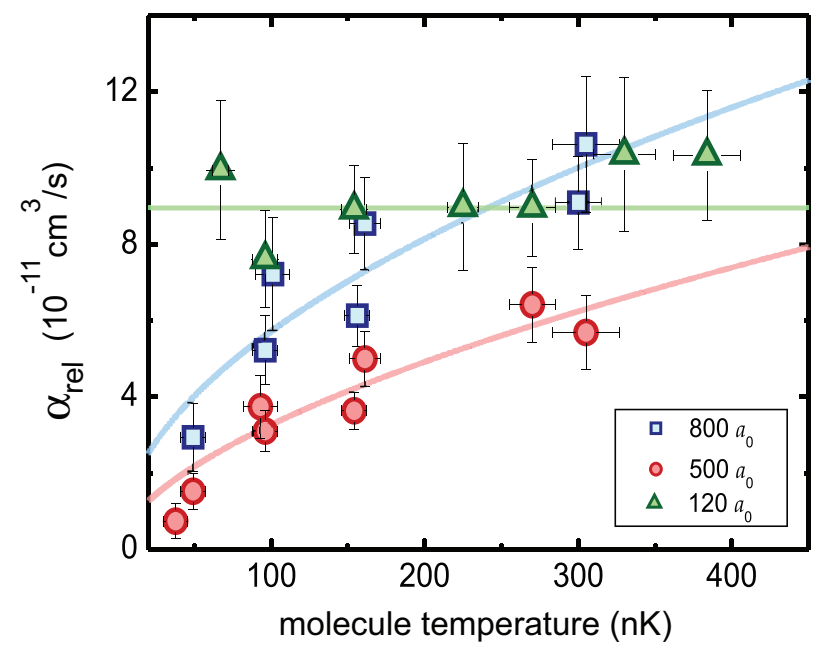

FIG. 4: (color online) Temperature dependence of the relaxation rate coefficient $\alpha_{\text {rel }}$ at $120 a_{0}$ (triangles), $500 a_{0}$ (squares), and $800 a_{0}$ (circles). The solid lines are introduced as guides to the eye.

observed a pronounced scattering length dependence, with a minimum in the loss coefficient as a most striking feature. We have also found that inelastic loss is further suppressed by decreasing the temperature. The existence of the minimum raises the question whether this feature can be understood in terms of universal four-body physics, similar to a minimum in three-body recombination at large positive scattering length [5, 7, 39, 40], which results from the destructive interference of two decay channels. The slow inelastic decay near the minimum may provide us with a favorable situation to study elastic dimer-dimer interactions or to search for universal four-body bound states [9].

We thank B. Esry, M. Baranov, D. Petrov, G. Shlyapnikov, and T. Köhler for fruitful discussions. We acknowledge support by the Austrian Science Fund (FWF) within SFB 15 (project part 16). S. K. is supported within the Marie Curie Intra-European Program of the European Commission. F. F. is supported within the Lise Meitner program of the FWF.

[1] A. S. Jensen et al., Rev. Mod. Phys. 76, 215 (2004).

[2] J. M. Blatt and V. F. Weisskopf, Theoretical Nuclear Physics (Wiley, New York, 1952).

[3] F. Luo et al., J. Chem. Phys. 98, 3564 (1993).

[4] W. Schöllkopf and J. P. Toennies, Science 266, 1345 (1994).

[5] E. Braaten and H.-W. Hammer, Phys. Rep. 428, 259 (2006).
[6] V. Efimov, Phys. Lett. B 33, 563 (1970).

[7] T. Kraemer et al., Nature 440, 315 (2006).

[8] M. T. Yamashita et al., Europhys. Lett. 75, 555 (2006).

[9] H. Hammer and L. Platter, Eur. Phys. J. A 32, 113 (2007).

[10] T. Köhler, K. Góral, and P. S. Julienne, Rev. Mod. Phys. 78, 1311 (2006).

[11] M. Inguscio, W. Ketterle, and C. Salomon, eds., Ultracold Fermi Gases (IOS Press, Amsterdam, 2008), Proceedings of the International School of Physics "Enrico Fermi", Course CLXIV, Varenna, 20-30 June 2006.

[12] D. S. Petrov, C. Salomon, and G. V. Shlyapnikov, Phys. Rev. Lett. 93, 090404 (2004).

[13] V. Efimov, Sov. J. Nuc. Phys. 29, 546 (1979).

[14] S. T. Thompson, E. Hodby, and C. E. Wieman, Phys. Rev. Lett. 94, 020401 (2005).

[15] M. Mark et al., Phys. Rev. A 76, 042514 (2007).

[16] C. Chin et al., Phys. Rev. A 70, 032701 (2004).

[17] T. Weber et al., Science 299, 232 (2003).

[18] The van der Waals length and energy can be defined as $r_{\mathrm{vdW}}=$ $\frac{1}{2}\left(m C_{6} / \hbar^{2}\right)^{1 / 4}$ and $E_{\mathrm{vdW}}=\hbar^{2} / m r_{\mathrm{vdW}}^{2}$, respectively [10].

[19] J. Herbig et al., Science 301, 1510 (2003).

[20] V. Milner et al., Phys. Rev. Lett. 86, 1514 (2001).

[21] N. Friedman et al., Phys. Rev. Lett. 86, 1518 (2001).

[22] A. Altmeyer et al., Phys. Rev. A 76, 033610 (2007).

[23] For the lowest temperature samples, the final time-averaged elliptic potential has trap frequencies of $10 \mathrm{~Hz}$ and $20 \mathrm{~Hz}$ in the horizontal plane, and $80 \mathrm{~Hz}$ in the vertical direction.

[24] The microwave drives the $|3,3\rangle \rightarrow|4,4\rangle$ hyperfine transition, while the resonant light pulse drives the closed optical transition $|4,4\rangle \rightarrow|5,5\rangle$, which pushes the atoms out of the optical trap. The purification is performed at a magnetic field of $35 \mathrm{G}$.

[25] G. Thalhammer et al., Phys. Rev. Lett. 96, 050402 (2006).

[26] S. Jochim et al., Phys. Rev. Lett 91, 240402 (2003).

[27] T. Köhler, E. Tiesinga, and P. S. Julienne, Phys. Rev. Lett. 94, 020402 (2005).

[28] E. Braaten and H. W. Hammer, Phys. Rev. A 70, 042706 (2004).

[29] J. P. D'Incao and B. D. Esry, Phys. Rev. Lett. 94, 213201 (2005).

[30] D. Petrov and G. Shyapnikov, private communication (2007).

[31] T. Mukaiyama et al., Phys. Rev. Lett. 92, 180402 (2004).

[32] N. Syassen et al., Phys. Rev. A 74, 062706 (2006).

[33] C. Chin et al., Phys. Rev. Lett. 94, 123201 (2005).

[34] S. Knoop et al., Phys. Rev. Lett. 100, 083002 (2008).

[35] Y. Inada et al., arXiv:0803.1405 (2008).

[36] L. D. Landau and E. M. Lifshitz, Quantum mechanics (Course of theoretical physics, Oxford: Pergamon Press, 1965).

[37] T. G. Lee et al., J. Chem. Phys. 125, 114302 (2006).

[38] G. Quéméner, N. Balakrishnan, and R. Krems, Phys. Rev. A 77, 030704(R) (2008).

[39] B. D. Esry, C. H. Greene, and J. P. Burke, Phys. Rev. Lett. 83, 1751 (1999).

[40] E. Nielsen and J. H. Macek, Phys. Rev. Lett. 83, 1566 (1999). 